- hier wiedergegebene - Eindruck der Beratungsperson mit den tatsächlichen Motiven der Mütter nicht übereinstimmt.

Im Ergebnis hat die vom Bundesministerium der Justiz durchgeführte Befragung von Jugendämtern und Rechtsanwält(inn)en ein sehr vielschichtiges Bild ergeben. Die Angaben der Teilnehmer(innen) beruhen ausschließlich auf Erinnerungswerten und Schätzwerten, nicht auf belastbaren statistischen Angaben.

Es ergibt sich hieraus, dass insbesondere die Einschätzung des EGMR, dass nicht immer kindbezogene Gründe der Anlass dafür sind, die Sorgeerklärung nicht abzugeben, insbesondere auf der Sicht der Väter beruhen und auf der persönlichen Einschätzung der Jugendamtsmitarbeiter(innen) und der teilnehmenden Rechtsanwält(inn)e(n). Eine echte Auseinandersetzung mit den Motiven hat nicht stattgefunden.

Auch der internationale Vergleich, der immer bemüht wird, führt hier nicht weiter. Die Antwort der Bundesregierung auf eine Anfrage der Grünen gibt an, dass die überwiegende Zahl der Rechtsordnungen in den Mitgliedstaaten der Europäischen Union den Eltern das Sorgerecht gemeinsam zuordne, und zwar kraft Gesetzes und unabhängig vom Personenstand der Eltern. ${ }^{9}$

Wie bereits der Richter am EGMR Schmitt in seinem Sondervotum zur Entscheidung richtig ausführt, gibt es einen europäischen Konsens zu der Frage nicht. Richtig ist, dass eine Vielzahl von europäischen Staaten die Entscheidung trifft, dass die elterliche Mitsorge des nichtehelichen Vaters entweder automatisch mit der Geburt - bzw. der Feststellung der Vaterschaft - entsteht und ein anderer Teil die Entscheidung trifft, dass der nichteheliche Vater jedenfalls per Antrag das Sorgerecht übertragen erhalten kann. So kennen beispielsweise Belgien, Bulgarien, Belarus, Tschechien, Ungarn, Polen, Russland, Slowenien, die Slowakei und die Ukraine die Mitsorge des nichtehelichen Vaters. Andere europäische Länder lassen die Mitsorge auf Antrag zu, wobei es auch hier gravierende Unterschiede gibt. Frankreich lässt den Antrag des nichtehelichen Vaters ausreichen, wenn er innerhalb des ersten Jahres nach der Geburt des Kindes gestellt wird. Andere Länder überprüfen die Situation der Eltern und des Kindes per Gericht, wenn die Mutter nicht zustimmt. Hier von einem europäischen Konsens zu sprechen, ginge sicher zu weit.

Im Gegensatz zu der Beendigung der Mitsorge bei ehelichen Kindern ist die Situation des nichtehelichen Vaters, der die Mitsorge begehrt, in der Regel völlig anders. Auch der Fall, den das EGMR zu entscheiden hatte, stellt nicht den Regelfall dar. Bei dem beantragenden Vater kann es sich auch um einen Vater handeln, der sich eben noch nicht als zuverlässiger Partner in Fragen um das Kind bewährt hat. Hier werden Väter zu finden sein, die keinen Unterhalt für das Kind zahlen, teilweise über Jahre hinweg, und die das Umgangsrecht mit dem Kind nicht regelmäßig wahrnehmen und trotzdem die rechtliche Verantwortung für das Kind mit übernehmen wollen. Hier werden Eltern aufeinandertreffen, die keinen Grundkonsens in Fragen der Erziehung haben. Eltern, die sich kaum kennen. Das Kind hat jedenfalls in jeder Situation einen Anspruch darauf, dass seine Eltern die für seine Zukunft wichtigen Fragen entscheiden. Sind die Eltern hierzu nicht in der Lage, wird diese Entscheidung nötigenfalls mit gerichtlicher Hilfe getroffen werden müssen. Gerichte werden dann gegebenenfalls in noch weiterem Umfang als heute entscheiden müssen, welcher Kindergarten für das Kind der richtige ist und welchen Namen das Kind tragen soll. Wenn aber das Gericht eine Entscheidung trifft, die dem Wunsch desjenigen widerspricht, bei dem das Kind lebt, muss dieser dann die Entscheidung des Gerichts tragen, weiter entwickeln und in der Zukunft befördern.

Ob dies der täglichen Realität in Familien entspricht und insbesondere dem Kindeswohl dienlich ist, wird die Frage sein, die der Gesetzgeber nun zu beantworten hat.

9 BT-Drs. 16/6078 vom 11.7.2007, <http://www.dnoti.de/DOC/2007/ BT_Drs_16_6078.pdf> (Zugriff 21.4.2010).

\title{
Die gleichgeschlechtliche Lebenspartnerschaft - Sorgerecht und Adoption
}

\author{
Daniela Helm \\ Rechtsreferendarin, Berlin
}

\section{Dr. Kerstin Niethammer-Jürgens}

Mitglied der Kommission Zivil-, Familien- und Erbrecht des djb; Rechtsanwältin, Potsdam

\section{Das Sorgerecht}

Das gemeinsame Sorgerecht der Eltern besteht auch nach Trennung und Aufnahme einer gleichgeschlechtlichen Le- benspartnerschaft fort. Die Homosexualität darf bei der Sorgerechtsentscheidung keine Rolle spielen, insbesondere darf sie nicht als negativ gewertet werden.

\section{Das „kleine Sorgerecht“}

Steht die elterliche Sorge beiden Elternteilen gemeinsam zu, zum Beispiel aufgrund einer zuvor bestandenen Ehe, wie es heute nach einer Scheidung der Regelfall ist, ist der (die) eingetragene Lebenspartner(in) nicht zur Ausübung des „kleinen Sorgerechts“ im Sinne von $\mathbb{\$} 9$ Abs. 1 LPartG befugt. Ist das 
Kind nicht aus einer Ehe hervorgegangen, hängt das Bestehen der gemeinsamen elterlichen Sorge davon $\mathrm{ab}$, ob eine Sorgeerklärung nach $\$ 1626 \mathrm{a}$ BGB abgegeben wurde. Ist eine solche Sorgeerklärung nicht abgegeben worden, besteht gem. \1626a Abs. 2 BGB Alleinsorge der Mutter kraft Gesetzes. Daher ist die Ausübung des „kleinen Sorgerechts“ in der Praxis meist für die eingetragene Lebenspartnerschaft lesbischer Paare relevant.

Steht dem in der gleichgeschlechtlichen Lebenspartnerschaft lebenden Elternteil das alleinige Sorgerecht nach gerichtlicher Entscheidung gem. \1671 BGB oder gesetzlich gem. $\ 1626$ a BGB zu, so kann der mit ihm in häuslicher Gemeinschaft lebende Partner (Stiefelternteil) nach $\mathbb{\int} 9$ Abs. 1 LPartG, in und ausschließlich bei Angelegenheiten des täglichen Lebens des Kindes mitentscheiden. Es ist jedoch immer zu bedenken, dass es sich gem. $\mathbb{\$} 9$ Abs. 1 LPartG um eine Befugnis zur Mitentscheidung, nicht zur Alleinentscheidung, handelt. Die Entscheidungen des Stiefelternteils müssen vom sorgeberechtigten Elternteil mitgetragen werden. Der hier verwendete Begriff „Angelegenheiten des täglichen Lebens" ist in der Bedeutung identisch mit der Legaldefinition aus $\mathbb{} 1687$ Abs. 1 S. 3 BGB. Nach $\mathbb{S} 1687$ Abs. 1 S. 3 BGB sind Entscheidungen in Angelegenheiten des täglichen Lebens in der Regel solche, die häufig vorkommen und die keine schwer abzuändernden Auswirkungen auf die Entwicklung des Kindes haben. Die Entscheidungsbefugnis nach $\mathbb{9}$ Abs. 1 LPartG des in häuslicher Gemeinschaft lebenden Lebenspartners des alleinsorgeberechtigten Elternteils wird als das „kleine Sorgerecht" bezeichnet. Zur Ausübung des sogenannten „kleinen Sorgerechts“ ist der Stiefelternteil nur befugt, wenn dessen Lebenspartner(in) Inhaber des alleinigen Sorgerechts ist.

Zur Ausübung des „kleinen Sorgerechts“ durch den Stiefelternteil bedarf es des Einvernehmens mit dem alleinsorgeberechtigten Elternteil. Dieses Einvernehmen ist durch den alleinsorgeberechtigten Elternteil jederzeit frei widerruflich. Leben die Lebenspartner nicht nur vorübergehend voneinander getrennt oder wurde die Lebenspartnerschaft gerichtlich aufgehoben oder besteht das alleinige Sorgerecht des Lebenspartners (der Lebenspartnerin) aus anderen Gründen nicht mehr, erlischt das „kleine Sorgerecht" des (der) nichtsorgeberechtigten Lebenspartners (Lebenspartnerin), da der Zweck des „kleinen Sorgerechts“ nicht mehr existent ist. Der Zweck des „kleinen Sorgerechts“ ist die Erleichterung von Problemen des alltäglichen Lebens aufgrund häuslicher Gemeinschaft des Lebenspartners (der Lebenspartnerin) mit dem Kind, die in solchen Fällen dann nicht mehr vonnöten ist.
Widerspricht die Ausübung des „kleinen Sorgerechts“ durch den (die) nichtsorgeberechtigte(n) Lebenspartner(in), der (die) weiterhin in häuslicher Gemeinschaft lebt, dem Kindeswohl, obliegt es dem Familiengericht, das „kleine Sorgerecht“ des Lebenspartners (der Lebenspartnerin) auszuschließen oder einzuschränken.

Eine Vertretung des Kindes ist gem. $\mathbb{S} 9$ Abs. 1 S. 2 LPartG jedoch ausgeschlossen, soweit nach \ 1795 BGB ein Vormund von der Vertretung ausgeschlossen wäre, da gem. $\mathbb{9}$ Abs. 1 S. 2 LPartG \ 1629 Abs. 2 S. 1 BGB entsprechend anzuwenden ist. Nach $\mathbb{S} 1795$ BGB ist eine Vertretung des Kindes dann ausgeschlossen, bei Rechtsgeschäften zwischen dem (der) Lebenspartner(in) bzw. seiner (ihrer) Verwandten in gerader Linie und dem Kind ( $\mathbb{S} 1795$ Abs. 1 Nr. 1 BGB), bei bestimmten, dem (der) Vertreter(in) zugute kommenden Rechtsgeschäften ( $\mathbb{S} 1795$ Abs. 1 Nr. 2 BGB), bei einem Rechtsstreit zwischen den in Nr. 1 genannten Personen und dem Kind bezüglich der in Nr. 2 genannten Rechtsgeschäfte sowie im Falle eines Insichgeschäftes nach $\mathbb{1} 181$ BGB ( $\mathbb{1 7 9 5}$ Abs. 2 BGB).

\section{Ist der (die) Lebenspartner(in) Inhaber der} alleinigen elterlichen Sorge, so ist seine (ihre) Lebenspartner(in) mit seiner (ihrer) Zustimmung zur Ausübung des sog. kleinen Sorgerechts berechtigt.

Das „kleine Sorgerecht“ ist keine Lebenspartnerschaftssache im Sinne von $\mathbb{2} 269$ FamFG, sondern eine Kindschaftssache nach $\mathbb{\$} 151$ Nr. 1 FamFG und somit eine Familiensache nach $\mathbb{S} 111$ Nr. 2 FamFG, auch wenn das Kind im gemeinsamen Haushalt der Lebenspartner lebt. Für Kindschaftssachen im Sinne von $\mathbb{1 5 1}$ Nr. 1 FamFG, also auch für Entscheidungen betreffend das „kleine Sorgerecht", ist das Familiengericht sachlich zuständig. Für Fälle mit Auslandsbezug richtet sich die internationale Zuständigkeit deutscher Gerichte für Kindschaftssachen nach $\mathbb{\int} 99$ FamFG. Gem. $\mathbb{9} 99$ FamFG ist ein deutsches Gericht in drei alternativen Fällen international zuständig: Erstens nach \ 99 Abs. 1 S. 1 Nr. 1 FamFG, wenn das Kind Deutsche(r) ist, wobei eine mehrfache Staatsangehörigkeit des (der) Minderjährigen die Zuständigkeitsregelung nicht ausschließt. Zweitens sind nach \ 99 Abs. 1 S. 1 Nr. 2 FamFG deutsche Gerichte international zuständig, wenn das Kind seinen gewöhnlichen Aufenthalt, also seinen Lebensmittel- 
punkt im Inland hat. Die Frage des gewöhnlichen Aufenthalts des Kindes im Herkunftsstaat ist unabhängig von der Staatsangehörigkeit des Kindes oder der Eltern zu klären. Die Zuständigkeit nach $\mathbb{\int} 99$ Abs. 1 S. 1 Nr. 2 FamFG ist gegenüber der Zuständigkeit nach $\mathbb{\int} 99$ Abs. 1 S. 1 Nr. 1 FamFG subsidiär. Die Zuständigkeit, welche sich aus dem Umstand ergibt, dass das Kind seinen gewöhnlichen Aufenthalt in Deutschland hat, gelangt folglich nur zur Anwendung, wenn das Kind nicht deutsche(r) Staatsbürger(in) ist. Die dritte Variante der internationalen Zuständigkeit deutscher Gerichte ist nach $\mathbb{S} 99$ Abs. 1 S. 2 FamFG gegeben, soweit das Kind der Fürsorge deutscher Gerichte bedarf. Diese Zuständigkeit ist wiederum subsidiär gegenüber den vorgenannten Zuständigkeiten. Also nur einschlägig, wenn das Kind weder die deutsche Staatsangehörigkeit besitzt, noch seinen gewöhnlichen Aufenthalt in Deutschland hat. Die örtliche Zuständigkeit richtet sich nach \152 FamFG. Die örtliche Zuständigkeit knüpft in $\mathbb{} 152$ Abs. 2 FamFG wieder an den gewöhnlichen Aufenthalt des Kindes an und in $\mathbb{S} 152$ Abs. 3 FamFG an das Bedürfnis der Fürsorge.

\section{Das Notsorgerecht}

In $\mathbb{9}$ Abs. 2 LPartG ist die sogenannte Notfallkompetenz geregelt. Bei Gefahr im Verzug ist der (die) Lebenspartner(in) des alleinsorgeberechtigten Elternteils (Stiefelternteil) dazu berechtigt, alle Rechtshandlungen vorzunehmen, die zum Wohl des Kindes notwendig sind, um einen dem Kind drohenden Schaden abzuwenden. Damit ist $\mathbb{9}$ Abs. 2 LPartG identisch mit $\mathbb{1 6 2 9}$ Abs. 1 S. 4 BGB. Der (die) Lebenspartner(in) muss den alleinsorgeberechtigten Elternteil von der Gefahr und den diesbezüglich getroffenen Maßnahmen unverzüglich, d.h. ohne schuldhaftes Zögern, unterrichten. Für die Ausübung des Notsorgerechts müssen die Voraussetzungen des $\mathbb{9}$ Abs. 1 LPartG nicht vorliegen.

Der (die) eingetragene Lebenspartner(in) muss für die Ausübung des Notsorgerechts nicht zur Ausübung des „kleinen Sorgerechts“ berechtigt sein. Er (sie) kann also auch dann Maßnahmen zum Schutz des Kindes ergreifen, wenn sein(e) (ihre) Lebenspartner(in) nicht Inhaber(in) der alleinigen elterlichen Sorge ist, sondern die gemeinsame elterliche Sorge mit dem anderen Elternteil des Kindes ausübt. Die zu ergreifenden Maßnahmen müssen aber aufgrund der Eilbedürftigkeit sofort notwendig sein und zwar in einer Dringlichkeit, die ein Abwarten auf die Zustimmung der sorgeberechtigten Elternteile unmöglich macht. Der Zweck des Notsorgerechts ist es, alle dem Kind drohenden Gefahren und dadurch entstehende Schäden schnellstmöglich und auf effektive Art und Weise abzuwenden. Damit dient das Notsorgerecht einzig dem Schutz des Kindeswohls.

Bei Streit über die Ausübung des Notsorgerechts zwischen dem (der) handelnden Lebenspartner(in) und dessen (deren) Lebenspartners (Lebenspartnerin) oder zwischen dem (der) handelnden Lebenspartner(in) und dem mitsorgeberechtigten Elternteil handelt es sich um eine Kindschaftssache im Sinne von $\mathbb{S} 151$ FamFG, da der Streitigkeit nicht die gemeinsame elterliche Sorge eines gemeinschaftlichen Kindes zugrunde liegt, wie dies $\$ 269$ Abs. 1 Nr. 3 FamFG erfordert. Bezüglich der internationalen, der örtlichen und der sachlichen Zuständigkeit gelten die Ausführungen zum „kleinen Sorgerecht“.

\section{Adoption}

Auch bei der Adoption darf die sexuelle Ausrichtung des (der) Annehmenden keine Rolle spielen. Die Homosexualität darf nicht negativ gewertet werden.

\section{Adoption mit Einwilligung des (der) anderen Lebenspartners (Lebenspartnerin)}

Gem. $\int 9$ Abs. 6 S. 1 LPartG ist es mit Einwilligung des (der) anderen Lebenspartners (Lebenspartnerin) möglich, ein Kind alleine zu adoptieren. Die Voraussetzung des Vorliegens einer Einwilligung des (der) eingetragenen Lebenspartners (Lebenspartnerin) dient der Integration des Kindes in die neue Familie. Das Kind soll nicht nur von einem (einer) Lebenspartner(in) gewollt sein, sondern der (die) andere Lebenspartner(in) muss diese Entscheidung zumindest billigen, um das Entstehen einer Familie möglich zu machen. Die Einwilligung kann aber auch gerichtlich ersetzt werden, wenn berechtigte Interessen des (der) anderen Lebenspartners (Lebenspartnerin) und der Familie des (der) Annehmenden nicht entgegenstehen, $\mathbb{S} 9$ Abs. 6 S. 2 LPartG i.V.m. $\mathbb{S} 1749$ Abs. 1 S. 2 und S. 3 BGB. Fraglich ist aber, ob die Ersetzung der Einwilligung nicht das Entstehen einer Familie unmöglich werden lässt. Akzeptiert der (die) andere Lebenspartner(in) die Entscheidung des (der) Lebenspartners (Lebenspartnerin), ein Kind als eigenes anzunehmen nicht, dann wird er (sie) auch das Kind nicht akzeptieren. In Fällen, in denen das Kind in den gemeinsamen Haushalt aufgenommen wird, scheint eine Ersetzung der Zustimmung dem Kindeswohl zu widersprechen. Der (die) Lebenspartner(in), der (die) das Kind schon von Beginn an nicht im Haushalt haben möchte, wird sich auch dem Entstehen einer Familie entgegensetzen. Die Einwilligung des (der) anderen Lebenspartners (Lebenspartnerin) darf dann nicht gerichtlich ersetzt werden, wenn er (sie) zur Abgabe aufgrund Geschäftsunfähigkeit außerstande ist oder sein (ihr) Aufenthalt dauernd unbekannt ist, $\mathbb{S} 9$ Abs. 6 S. 2 LPartG i.V.m. $\$ 1749$ Abs. 3 BGB. In der Praxis ist jedoch eine solche Adoption äußerst schwer zu erreichen, obwohl es vorgesehen ist, dass die sexuelle Ausrichtung der Antragsteller keine Rolle spielt. Es existieren weit mehr Adoptionsanträge als es zur Adoption freigegebene Kinder gibt. In der Regel wird ein Elternteil, das in „normaler“ Ehe lebt, gegenüber dem in gleichgeschlechtlicher Lebensgemeinschaft lebenden Elternteil bevorzugt, obwohl die sexuelle Ausrichtung des (der) Annehmenden keinen Ausschlag geben darf.

Die Adoption durch eine(n) Lebenspartner(in) im Sinne von $\mathbb{9}$ Abs. 6 LPartG ist eine Lebenspartnerschaftssache

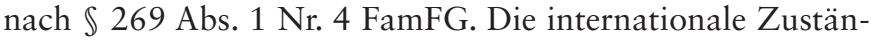
digkeit deutscher Gerichte für Lebenspartnerschaftssachen richtet sich nach $\mathbb{} 103$ FamFG. Nach $\mathbb{} 103$ Abs. 3 FamFG ist für diese Adoptionssachen $\mathbb{\$} 101$ FamFG entsprechend anzu- 
wenden. Deutsche Gerichte sind international zuständig, wenn der (die) Annehmende oder das Kind deutsche $(r)$ Staatsangehörige $(r)$ ist $(\mathbb{S} 101 \mathrm{Nr} .1$ FamFG) oder seinen (ihren) gewöhnlichen Aufenthalt im Inland hat ( $\$ 101$ Nr. 2 FamFG). Folglich sind deutsche Gerichte stets zuständig, wenn das anzunehmende Kind die deutsche Staatsangehörigkeit besitzt oder sein gewöhnlicher Aufenthalt in Deutschland liegt, unabhängig von der Staatsangehörigkeit oder des gewöhnlichen Aufenthalts des (der) Annehmenden. Dabei ist es ausreichend, dass das Kind zumindest auch die deutsche Staatsangehörigkeit besitzt, eine mehrfache Staatsangehörigkeit steht der internationalen Zuständigkeit deutscher Gerichte nicht entgegen (Art. 5 Abs. 1 S. 2 EGBGB). Dies gilt auch für den Fall, dass nur der (die) Annehmende die deutsche Staatsangehörigkeit besitzt oder sein (ihr) gewöhnlicher Aufenthalt im Inland liegt. Die örtliche Zuständigkeit be-

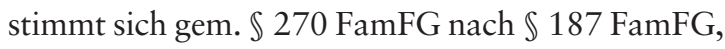
da auf $\mathbb{S} 111$ Nr. 4 FamFG (Adoptionssachen) verwiesen wird und diesbezüglich die jeweils geltenden Vorschriften entsprechend anzuwenden sind. Für Verfahren betreffend die Annahme als Kind ( $\mathbb{S}$ 186 Nr. 1 FamFG) und die Ersetzung der Einwilligung zur Annahme als Kind ( $\mathbb{S} 186$ Nr. 2 FamFG) ist gem. $\ 187$ Abs. 1 FamFG das Gericht örtlich zuständig, in dessen Bezirk der (die) Annehmende seinen (ihren) gewöhnlichen Aufenthalt hat. Besteht kein inländischer gewöhnlicher Aufenthalt des (der) Annehmenden, ist nach $\mathbb{} \int 187$ Abs. 2 FamFG das Gericht örtlich zuständig, in dessen Bezirk das anzunehmende Kind seinen gewöhnlichen Aufenthalt hat. $\int 187$ Abs. 4 FamFG regelt den Fall, wenn für diese Verfahren ausländische Sachnormen zur Anwendung gelangen (nur für Anerkennungs-, Feststellungs- und Umwandlungsverfahren ist das AdWirkG einschlägig). Dann gilt $\mathbb{} 5$ Abs. 1 S.1 und Abs. 2 AdWirkG entsprechend. Dies führt zu einer Zuständigkeitskonzentration bei den Familiengerichten am Sitz des OLG. Subsidiär, d.h. nur wenn keine der vorgenannten Zuständigkeitsregelungen greift, ist das Amtsgericht Schöneberg in Berlin örtlich zuständig. Die Anwendung materiellen Sachrechts für die Adoption richtet sich nach Art. 22 EGBGB. Nach Art. 22 Abs. 1 EGBGB unterliegt die Annahme dem Recht des Staates, dem der (die) Annehmende bei der Annahme angehört. Sachlich zuständig ist das Familiengericht, da Adoptionssachen nach $\mathbb{S} 111$ Nr. 4 Familiensachen sind.

\section{Stiefkindadoption}

\ 9 Abs. 7 LPartG ermöglicht es dem (der) Lebenspartner(in), das Kind seines (ihres) Le- benspartners (Lebenspartnerin) anzunehmen. Nur so können gleichgeschlechtliche Lebenspartner ein gemeinsames Kind großziehen in Ausübung der gemeinsamen elterlichen Sorge, $\mathbb{S} \mathbb{S} 9$ Abs. 7 S. 2 LPartG, 1754 Abs. 3 BGB. Adoptiert der (die) andere Lebenspartner(in) das leibliche Kind seines (ihrer) Lebenspartners (Lebenspartnerin), erlischt das Verwandtschaftsverhältnis zu dem vorherigen Elternteil, inklusive des Verwandtschaftsverhältnisses zu dessen Verwandten, $\mathbb{S} \mathbb{S} 9$ Abs. 7 S. 2 LPartG, 1755 Abs. 2 BGB. Hat der (die) eingetragene Lebenspartner(in) das Kind zuvor gem. $\mathbb{9}$ Abs. 6 LPartG allein angenommen, kann eine Stiefkindadoption nicht erfolgen.

\section{Derzeit ist die Stiefkindadoption die einzige Möglichkeit für eingetragene Lebenspartner(innen), in Ausübung der gemeinsamen elterlichen Sorge ein Kind erziehen.}

Voraussetzung für die Stiefkindadoption ist es, dass es sich um die Annahme eines leiblichen Kindes des (der) Lebenspartners (Lebenspartnerin) handelt. Eine weitere Voraussetzung ist die Zustimmung oder das Fehlen des anderen rechtlichen Elternteils. So kann das während des Bestandes der eingetragen Lebenspartnerschaft geborene Kind, etwa durch künstliche Befruchtung (heterologe Insemination), gemeinsames Kind der Lebenspartner(innen) werden, wenn eine Adoption durch den (die) andere(n) Lebenspartner(in) nach $\mathbb{} 9$ Abs. 7 LPartG erfolgt. Ein Pendant zur Regelung des \ 1592 Nr. 1 BGB existiert für die eingetragene Lebenspartnerschaft nicht. Nach $\mathbb{1 5 9 2}$ Nr. 1 BGB gilt als Vater des Kindes der Mann, der zum Zeitpunkt der Geburt mit der Mutter des Kindes verheiratet ist. Ein Verwandtschaftsverhältnis kraft Gesetz für den (die) eingetragene(n) Lebenspartner(in) sieht das Gesetz nicht vor. Auch eine analoge Anwendung des $\mathbb{1 5 9 2}$ Nr. 1 BGB kommt mangels Vergleichbarkeit der Sachverhalte nicht in Betracht. Wird das Kind während des Bestandes einer eingetragenen Lebenspartnerschaft geboren, bedarf es für die Stiefkindadoption lediglich der Zustimmung der Mutter des Kindes, da kein anderer rechtlicher Elternteil vorhanden ist. Ist das Kind während einer vorausgegangen Ehe geboren oder hat der Vater die Vaterschaft anerkannt, bedarf es dessen Zustimmung. 
Die Stiefkindadoption darf nicht der Verfestigung der Lebenspartnerschaft dienen, sondern soll einzig die Rechte des Kindes verbessern, etwa dadurch, dass mehrere Unterhaltspflichtige existieren. Die Adoption muss das Kindeswohl verbessern bzw. fördern. Die Regelung der Stiefkindadoption dient somit einzig dem Kindeswohl und unterliegt dem in \1741 Abs. 1 BGB normierten Grundsatz.

Der zentrale Punkt ist, dass das Kindeswohl das entscheidende Kriterium der Adoption durch den (die) Lebenspartner(in) sein muss. Lehnt das Kind selbst die Annahme durch den (die) Lebenspartner(in) seines Elternteils ab, ist eine Adoption nicht möglich, denn durch die Adoption muss die Entstehung eines Eltern-Kind-Verhältnis zu erwarten sein. Die Vorschriften der $\mathbb{S} 1741 \mathrm{ff}$. BGB, insbesondere die Vorschriften über die Einwilligung $\mathbb{S} \mathbb{S} 1746 \mathrm{ff}$. BGB gelten auch hier, obwohl sie nicht ausdrücklich in $\mathbb{S}$ Abs. 7 S. 2 LPartG genannt sind. Der annehmende Lebenspartner muss gem. $\mathbb{S} 9$ Abs. 7 S. 2 LPartG i.V.m. $\mathbb{S} 1741$ Abs. 2 S. 3, 1743 S. 1 BGB das 21. Lebensjahr vollendet haben. Nach $\mathbb{\int} 9$ Abs. 7 S. 2 LPartG gelten $\mathbb{S} 1751$ Abs. 2 und Abs. 4 S. 2 BGB. Danach sind die Lebenspartner dem Kind vorrangig vor anderen Verwandten zur Gewährung von Unterhalt verpflichtet, bereits ab dem Zeitpunkt, an dem die erforderliche Einwilligung der Elternteile erteilt wurde und das Kind sich in der Obhut der Lebenspartner befindet. Die tatsächliche Adoption muss noch nicht erfolgt sein.

Bei der Stiefkindadoption lebt das Kind regelmäßig bereits vor Antragstellung im gemeinsamen Haushalt der Lebenspartner. Die Annahme als Kind wird auf Antrag des (der) Annehmenden vom Vormundschaftsgericht in Form eines Beschlusses erteilt. Durch den Beschluss werden die Rechte und Pflichten des (der) Lebenspartners (Lebenspartnerin), der (die) bereits Elternteil des Kindes ist (und auch bleibt), nicht berührt. Nach $\mathbb{9}$ Abs. 7 S. 2 LPartG i.V.m. \ 1754 Abs. 1 und Abs. 3 BGB erlangt das Kind durch die Annahme die Rechtsstellung eines gemeinsamen Kindes der Lebenspartner, denen sodann die elterliche Sorge gemeinsam zusteht. Mit der Annahme erlöschen gem. $\mathbb{S}$ Abs. 7 S. 2 LPartG i.V.m. $\mathbb{1 7 5 5}$ Abs. 2 BGB das Verwandtschaftsverhältnis - inklusive der daraus resultierenden Rechte und Pflichten - des Kindes zu dem anderen Elternteil und dessen Verwandten. Eine Ausnahme bildet $\$ 9$ Abs. 7 S. 2 LPartG i.V.m. $\mathbb{1 7 5 6}$ Abs. 2 BGB, wonach das Verwandtschaftsverhältnis zu den Verwandten des anderen Elternteils nicht durch die Annahme erlischt, wenn der andere Elternteil zuvor verstorben ist und die elterliche Sorge vor dem Tod gemeinsam bestand. Durch die Annahme erhält das Kind den gemeinsamen Namen der Lebenspartnerschaft; existiert ein solcher nicht, richten sich die namensrechtlichen Folgen nach $\mathbb{\$} 9$ Abs. 7 S. 2 LPartG i.V.m. $\mathbb{1 7 5 7}$ Abs. 2 S. 1 BGB. Es kann vor dem Ausspruch der Annahme entweder der Name des (der) Lebenspartners (Lebenspartnerin), der (die) bereits Elternteil ist, gewählt werden oder der Name des (der) Annehmenden. Nach $\mathbb{S} 9$ Abs. 7 S. 2 LPartG i.V.m. \ 1772 Abs. 1 S. 1 lit. c BGB ist auch die Stiefkindadoption eines Erwachsenen möglich, mit denselben Wirkungen der Minderjährigenadoption.

Betreffend die elterliche Sorge gem. $\int 269$ Abs. 1 Nr. 3 FamFG ist nur die gemeinsame elterliche Sorge erfasst, nicht das sich vom (von der) Lebenspartner(in) abgeleitete „kleine Sorgerecht". Ist also eine Stiefkindadoption erfolgt und besteht Streitigkeit bezüglich der hierdurch entstandenen gemeinsamen elterlichen Sorge, fallen diese Verfahren unter den Begriff der Lebenspartnerschaftssachen im Sinne des $\mathbb{S} 269$ Abs. 1 Nr. 3 FamFG. Jedoch verweist $\$ 270$ Abs. 1 FamFG auf \$111 Nr. 2 FamFG (Kindschaftssachen), so dass bezüglich der örtlichen und sachlichen Zuständigkeit die Ausführungen zum „kleinen Sorgerecht“ gelten.

Die Stiefkindadoption selbst (sowie die Ersetzung der Einwilligung) nach $\mathbb{S}$ Abs. 7 LPartG kann keine Lebenspartnerschaftssache im Sinne von $\mathbb{} 269$ Abs. 1 Nr. 4 FamFG sein, wenn zum Zeitpunkt der Stiefkindadoption noch ein Verwandtschaftsverhältnis zu dem anderen leiblichen Elternteil besteht. Dieser Elternteil kann ja nicht Beteiligter einer Lebenspartnerschaftssache sein. Daher ist die Behandlung als Adoptionssache im Sinne von $\mathbb{S} 186$ FamFG gerechtfertigt. Das Verfahren richtet sich folglich nach den $\$ \mathbb{S} 186 \mathrm{ff}$. FamFG. Besteht kein Verwandtschaftsverhältnis zu einem anderen Elternteil, der nicht der Lebenspartnerschaft angehört, sind über S $\int 270$ Abs. 1 i.V.m. $\mathbb{1} 11$ Nr. 4 FamFG die Vorschriften der Adoption, die $\mathbb{S} \mathbb{S} 186$ ff. FamFG anzuwenden. Diesbezüglich wird auf die Ausführungen unter „Adoption mit Einwilligung des anderen Lebenspartners" verwiesen.

\section{Adoption durch beide Lebenspartner}

Eine solche Regelung existiert nicht. Eine gemeinschaftliche Adoption fremder Kinder durch gleichgeschlechtliche Lebenspartner ist nicht möglich. Im Gegensatz zu Ehepartnern können gleichgeschlechtliche Lebenspartner durch eine gemeinsame Adoption kein gemeinschaftliches Kind großziehen. Diese Wertung ist jedoch nicht nachvollziehbar. Das Aufwachsen eines Kindes in gleichgeschlechtlicher Lebenspartnerschaft schadet dem Kindeswohl nicht. Warum sollte kein rechtliches Elternverhältnis durch gemeinsame Adoption eines „fremden" Kindes herstellbar sein, so wie es die Konstellation der nachfolgend dargestellten Stiefkindadoption ermöglicht. In einigen Ländern (wie zum Beispiel in Schweden, in den autonomen spanischen Gebieten, in den Niederlanden) ist die gemeinsame Adoption „fremder“ Kinder bereits möglich. Selbst nach der letzten Neufassung des $\mathbb{9}$ LPartG ist eine solche Regelung nicht eingefügt worden. Einer Adoption durch beide Lebenspartner steht die Wertung des Art. 6 Abs. 1 GG nicht entgegen. Vielmehr wäre es doch im Sinne der Gleichstellung von Nöten das Recht, welches einem Ehepaar zugestanden wird ( $\$ 1741$ Abs. 2 S. 2 BGB), auch den gleichgeschlechtlichen Lebenspartnern nicht zu verwehren. Würde ein solches Recht auch der eingetragenen Lebenspartnerschaft zugestanden, wäre dies ein weiterer großer Schritt in Richtung der Gleichstellung beider Lebensformen. Betrachtet man die geschichtliche Entwicklung des Institutes der gleichgeschlecht- 
lichen Lebenspartnerschaft, so wird man wohl davon ausgehen können, dass sich aufgrund dieser Entwicklung die Rechtsstellung der gleichgeschlechtlichen Lebenspartner im Laufe der Zeit weiter verbessern wird.

\section{Fazit}

Dem (der) gleichgeschlechtlichen Lebenspartner(in) obliegt das Sorgerecht bzw. dessen Ausübung folglich nur in Fällen, in denen sein(e) (ihr(e) eingetragene(r) Partner(in) alleinsorgeberechtigt ist, in Form des sogenannten „Kleinen Sorgerechts“, \9 Abs. 1 LPartG, oder bei Gefahr im Verzug in Form des Notsorgerechts, $\mathbb{S} 9$ Abs. 2 LPartG. Die gemeinsame elterliche Sorge können gleichgeschlechtliche Lebenspartner nur dann ausüben, wenn der (die) eine Lebenspartner(in) das Kind des (der) anderen adoptiert, $\mathbb{S} 9$ Abs. 7 LPartG, also in Form der sogenannten Stiefkindadoption. Ob der Gesetzgeber in $\mathrm{Zu}-$ kunft auch gleichgeschlechtlichen Lebenspartnern die gemeinschaftliche Adoption fremder Kinder gestattet, wie dies bereits in einigen Ländern der Fall ist, und damit die Rechte der homosexuellen Beziehungen weiter stärkt, bleibt abzuwarten.

\section{Literatur}

Bruns, Manfred/Kemper, Rainer (Hrsg.), Lebenspartnerschaftsrecht, Handkommentar, 2. Auflage, § 9, Baden-Baden 2005.

Frederici, Peter/Kemper, Rainer (Hrsg.), Familienverfahrensrecht, Nomos Handkommentar, Baden-Baden 2009; Kemper § 99, §101, §103, § 111; Fritsche $\S 186,187$; Kemper § 269, § 270.

Gerhard, Kirsten S., Die eingetragene Lebenspartnerschaft - eine historisch-dogmatische Bestandsaufnahme zur Frage nach einem neuen familienrechtlichen Institut, Göttingen 2009, $66 \mathrm{ff}$.

Muscheler, Karlheinz, Das Recht der eingetragenen Lebenspartnerschaft, Handbuch für die gerichtliche, anwaltliche und notarielle Praxis, 2. Aufl., Berlin 2004; 5.9 Lebenspartnerschaft und Kinder, $331 \mathrm{ff}$.

Prütting, Hanns/Helms, Tobias (Hrsg.), FamFG Kommentar, Köln 2009; Hau $\S 99, \S 101, \S 103$; Helms $\S 111$; Krause $\S 186,187$; Heiter $\S 269$, §270.

Rauscher, Thomas (Hrsg.), Münchener Kommentar zur Zivilprozessordnung: ZPO, Band 4: FamFG, 3. Aufl., München 2010; Rauscher § 99,

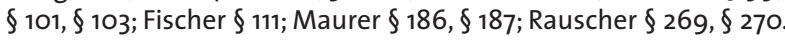

Säcker, Franz Jürgen/Rixecker, Roland (Hrsg.), Münchener Kommentar zum Bürgerlichen Gesetzbuch: BGB, C.H. Beck Verlag (oder München plus Jahr ergänzen); Maurer, $\$ \S 1741$ ff.; Dethloff, ZPR 2004, 195

\section{Französische Feministinnen streiten wieder}

\section{Buchrezension zu Elisabeth Badinters „Le Conflit, la femme et la mère“}

Badinter, Élisabeth: Le conflit, la femme et la mère. 270 Seiten, broschiert, EAN : 9782081231443 Flammarion 2010.

\section{Elisabeth Badinter}

Le conflit

la femme et la nuère

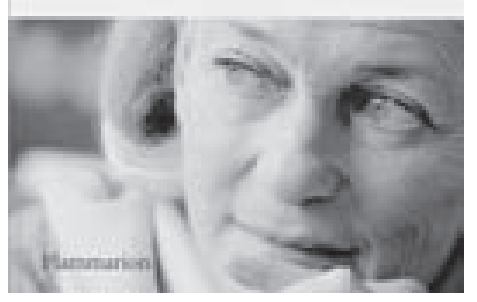

Es ist nicht das erste Mal, dass die Literaturwissenschaftlerin und feministische Philosophin Elisabeth Badinter (Jahrgang 1944) mit einem Buch eine heftige Debatte um die Rolle der Frau und den französischen Feminismus entfacht. Anfang Februar kam ihr letztes Buch („,Le Conflit") auf den Markt ${ }^{1}$ und wird seitdem in sämtlichen Medien kontrovers diskutiert; dank ihres Buches erfahren der verstaubte französische Feminismus und die Diskussion um die Rolle der Frau/Mutter in der französischen Gesellschaft neue Impulse. Kein Wunder, dass die Reaktionen sehr hitzig sind, denn Badinter greift Stillverfechterinnen, die neuen französischen „Ökos”, Kinderpsychiater(innen), Hebammen und Ärzt(innen) und nicht zuletzt junge Mütter an und macht sie alle für den schleichenden Verfall emanzipatorischer Errun- genschaften in Frankreich mitverantwortlich. Der ca. 250 Seiten umfassende Essay liest sich wegen des Badinter eigenen Schreibstils leicht, ist mit vielfältigen Verweisen und statistischem Unterbau versehen und möchte eher einen (populär-) wissenschaftlichen als politischen Eindruck machen.

Vielleicht liegt es daran, dass ich mich selbst als Mutter einer kleinen Tochter vom Thema „Rollenkonflikt” angesprochen fühle - jedenfalls habe ich in den vergangenen fünf Jahren keine so vehemente und vielschichtige Diskussion um feministische Thesen in Frankreich verfolgt. Grund genug, dies mit den deutschen Juristinnen zu teilen, zumal die moralisierende Familiendiskussion in Deutschland von Badinter in ihrem Buch wiederholt als Negativbeispiel und Ursache für die niedrige Geburtenrate einerseits und eine Emanzipationsbremse der deutschen Frauen andererseits herangezogen wird.

Die Autorin beginnt ihr Essay mit einer entspannenden Demystifizierung der „perfekten” Mutter, die es ihrer Meinung nach nicht gibt und auch nicht geben muss.

Im Kern attackiert sie die Renaissance eines „Naturalismus“, der sich seit einigen Jahren in der französischen Gesellschaft breit zu machen scheint. Dieser geht einher mit einem „radikalen Ökologieverständnis”, welches die französischen Mütter mit Windelwaschen, der Jagd nach den besten Biopro-

1 Nach Angaben des Flammarion Verlages ist ein Übersetzung und Publikation im Beck-Verlag in Verhandlung. 